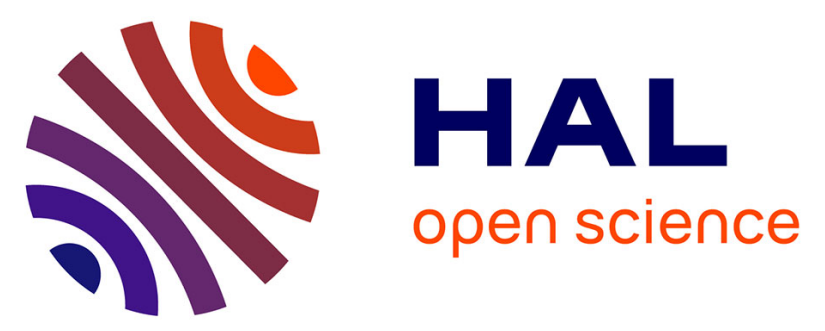

\title{
Fonctionnement hydrique de sols à argile gon- flante cultivés. I. - Analyse des influences du travail du sol et de l'irrigation sur le fonctionnement hydrique de sols à argile gonflante dans des exploitations de Guadeloupe (F.W.I.)
}

Bertrand Ney, J. André, N. Jean-Bart, A. Mulciba

\section{To cite this version:}

Bertrand Ney, J. André, N. Jean-Bart, A. Mulciba. Fonctionnement hydrique de sols à argile gonflante cultivés. I. - Analyse des influences du travail du sol et de l'irrigation sur le fonctionnement hydrique de sols à argile gonflante dans des exploitations de Guadeloupe (F.W.I.). Agronomie, 1987, 7 (4), pp.247-256. hal-00884989

\section{HAL Id: hal-00884989 https://hal.science/hal-00884989}

Submitted on 1 Jan 1987

HAL is a multi-disciplinary open access archive for the deposit and dissemination of scientific research documents, whether they are published or not. The documents may come from teaching and research institutions in France or abroad, or from public or private research centers.
L'archive ouverte pluridisciplinaire HAL, est destinée au dépôt et à la diffusion de documents scientifiques de niveau recherche, publiés ou non, émanant des établissements d'enseignement et de recherche français ou étrangers, des laboratoires publics ou privés. 


\title{
Fonctionnement hydrique de sols à argile gon- flante cultivés. I. - Analyse des influences du tra- vail du sol et de l'irrigation sur le fonctionne- ment hydrique de sols à argile gonflante dans des exploitations de Guadeloupe (F.W.I.)
}

\author{
Bertrand NEY \\ avec la collaboration technique de J. André, N. Jean-Bart et A. Mulciba \\ I.N.R.A., Station d'Agronomie - Science du sol, Centre de Recherches des Antilles et de la Guyane, \\ B.P. 1232 - 97184 Pointe-à-Pitre, Guadeloupe.
} est analysé pour différents régimes pluviométriques.

Mots clés additionnels : Sol, toposéquence, argile gonflante, fonctionnement hydrique, tomate, travail du sol, irrigation. behaviour of swelling soils.

Highly swelling clay soils in Guadeloupe were shown to occur in rather heterogeneous soil sequences, including both very deep clayey and shallow stony profiles. The high amounts of smectite clay mineral in vertisols were the cause of their highly constraining physical properties. Under natural savannah cover, an extreme bimodal pore distribution was responsible for a characteristic twofold water infiltration pattern : rapid fill-up of the large soil cracks and a very slow diffusion into the microporous soil peds. During filling up, soil cracks progressively closed at the soil surface, and the infiltration rates strongly decreased. However soil tillage changed this behaviour, in particular soil porosity in the tilled layer generally increased. In some cases, an impermeable ploughed pan was created causing a rupture of the soil cracks. This ruptured to decreased internal drainage. The appearance of a perched water table on the pan limited vertical root proliferation. The shallow stony soils were freely drained but water availability was a limiting factor. The effect of different irrigation doses on the response ' of deep clayey and shallow stony ploughed soils under tomato cover is discussed.

Additional key words : Soil, toposequence, swelling clay, hydric behaviour, tomato, tillage, irrigation. 


\section{INTRODUCTION}

La fertilité chimique des sols à argile gonflante (FINCK et YeNKATESWARLU, 1982) incite à les mettre en valeur de manière intensive. Cependant, leur occurrence naturelle sous climat à saison sèche marquée ou très marquée rend l'irrigation indispensable. De nombreux auteurs soulignent les difficultés de leur mise en culture (JEWITT et al., 1979 ; VIRMANI et al., 1982 ; WARKENTIN, 1982). Celles-ci proviennent en grande partie du faible drainage interne des sols profonds (vertisols proprement dits), lorsqu'ils sont saturés.

Des travaux menés en pays tempérés sur des sols dont la teneur en argile est moins extrême font état de l'importance du maintien de la continuité des pores de grande taille, dits de "transit», pour le passage de l'eau dans les couches profondes et l'aération du sol (Goss et al., 1978 ; Goss et al., 1984). Dès lors, on peut supposer que l'occupation des vertisols par des cultures à cycle court, pour lesquelles le travail du sol et l'irrigation sont fréquents entraîne une gestion délicate de leur fonctionnement hydrique.

Les conditions sont réunies en Grande Terre de Guadeloupe pour étudier ce fonctionnement :

- les sols sont toujours très argileux avec plus de 80 p. 100 de smectites calciques dans la terre totale,

- le fonctionnement hydrique des vertisols est bien connu (JAILLARD et CABIDOCHE, 1984),

- les cultures maraîchères, irriguées, dont les successions sont rapides, connaissent un développement important (PELlerin et NEY, 1985 ; NEY, 1985).

La diversité des situations offre un terrain expérimental présentant le schéma factoriel suivant :

- les sols à argile gonflante sont développés en toposéquences allant du sol lithomorphe argilocaillouteux peu profond (position haute convexe) au vertisol argileux profond à tendance hydromorphe (position basse concave). Le calcaire sous-jacent est généralement très poreux et très friable (CABIDOCHE, 1986),

- les régimes d'irrigation sont très variés,

- l'itinéraire technique de préparation du sol est unique et consiste en deux passages de charrue à disques,

- enfin la tomate, fortement représentée dans les successions, est une plante test adaptée à la mise en évidence des accidents par asphyxie.

Cet article s'attachera à l'étude par voie d'enquêtes dans les exploitations maraîchères des perturbations apportées au fonctionnement hydrique des sols à argile gonflante par le travail du sol et l'irrigation.

\section{MATÉRIEL ET MÉTHODE}

\section{A. Matériel}

\section{Fonctionnement hydrique d'un sol à argile gon- flante en condition naturelle}

Les vertisols sur calcaire corallien de Guadeloupe contiennent de 70 à $80 \%$ de smectites par rapport à la terre totale. Sous savane spontanée, JAILLARD et CABIDOCHE (1984) ont montré la liaison étroite existant entre les dynamiques hydrique et porale : lorsque les vertisols ne sont pas saturés, la mégaporosité fissurale est responsable de l'infiltratibilité élevée. Des zones humides profondes en bulbes se développent à son voisinage. Ceux-ci contrastent avec les massifs interfissuraux, à porosité fine et faible infiltrabilité. Puis le gonflement superficiel des massifs entraîne une fermeture des fissures ; dès lors l'infiltration est très faible, interdisant le plus souvent une réhumectation profonde des massifs. Ces sols conservent la plupart du temps des champs d'humidité très hétérogènes, mémoire de l'infiltration privilégiée par les fissures. Par ailleurs, les fentes se situent préférentiellement sous les dépressions du micro-relief lorsque celui-ci existe; le microruissellement augmente alors le flux rentrant dans ces zones, accentuant ainsi l'organisation et le contraste de l'hétérogénéité hydrique (fig. 1).

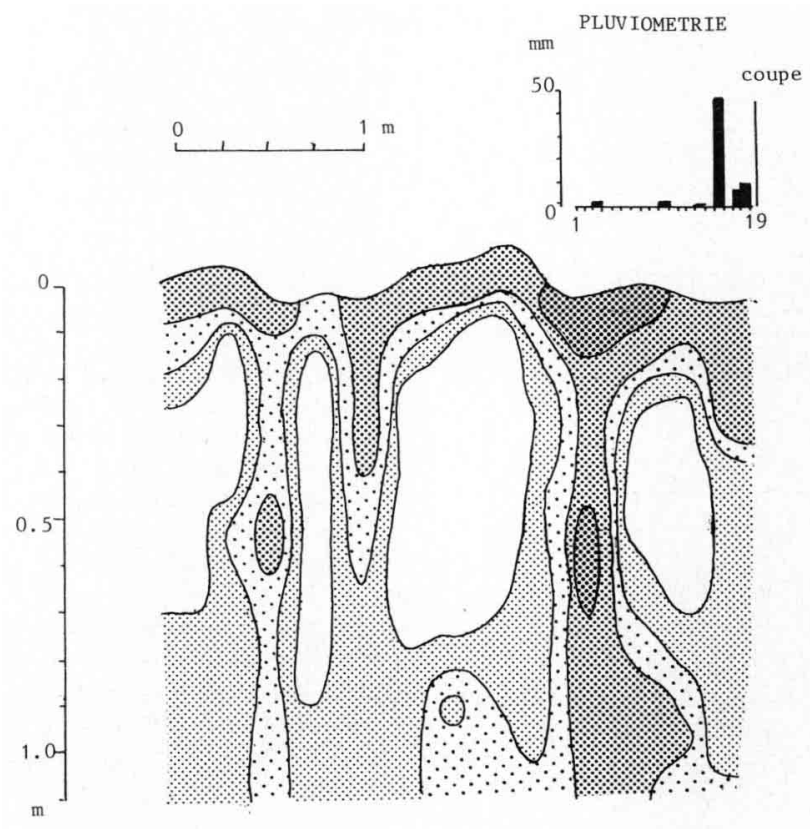

Figure 1

Un exemple de très fort contraste d'humidité après une pluie soulignant l'infiltration par les fentes de retrait dans un vertisol sous savane spontanée.

An example of water content contrast after rain, showing infiltration through the cracks in a vertisol.

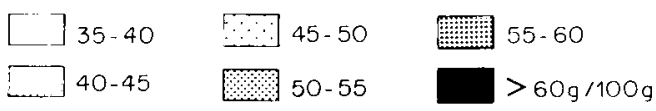

Dans les sols lithomorphes, la liaison entre dynamique hydrique et porale s'estompe. Lors du retrait, les éléments grossiers constituent des zones de fissuration isotrope, favorisant les transferts d'eau dans le profil et une homogénéisation des humidités. De plus, leur situation en topographie convexe assure l'évacuation latérale rapide de l'eau en excès.

\section{Dispositif}

La Tomate (Lycopersicon esculentum, var. Tropic) a été choisie en fonction de son extension régionale et pour sa sensibilité aux conditions de milieu, notamment aux excès d'eau. Sept parcelles ont été choisies 
dans six exploitations de la Grande Terre de Guadeloupe. Sur chacune d'elles, ont été sélectionnées 2 ou 3 placettes choisies en fonction des caractéristiques du sol, proximité du calcaire et taux d'éléments grossiers, mesurés par la méthode de la résistivimétrie (CABIDOCHE, 1986). Elles comportent 20 plantes. La figure 2 représente la position des placettes sur la coupe d'une toposéquence-type. Celles-ci seront identifiées tout au long du texte par un symbole X Ym, où X est l'identificateur de la parcelle, $Y$ le type de sol ( $Y$ pour vertisol de dépression, I pour vertisol de pente, $C$ pour les sols lithomorphes), et $m$ différenciant 2 placettes au type de sol identique. Le mode de conduite, assez uniforme, est résumé dans le tableau 1 . La préparation du sol est commune à toutes les parcelles. L'itinéraire technique de préparation du sol consiste en 2 passages de charrue à disques successifs suivis d'un sillonnage. Les plants sont placés au fond du sillon, tous les 0,30 à $0,35 \mathrm{~cm}$ sur les rangs distants de $1,60 \mathrm{~m}$. Toutes les parcelles sont arrosées au moyen d'asperseurs de type « sprinklers ». Les doses d'arrosage ont été relevées en plaçant un pluviomètre sur chacune des placettes. Doses d'arrosage et ETP cumulées mois par mois à partir de la plantation figurent dans le tableau 2. L'ETP est calculée à partir du Rayonnement global au moyen d'une • relation linéaire mise au point par $\mathrm{SCHOCH}$ et al.
(1975). Cette valeur variant peu (de 128 à $155 \mathrm{~mm}$ ) pendant la durée de l'expérimentation, nous avons choisi d'exprimer les pluviométries en multiples d'ETP.

\section{B. Méthodes}

La convergence éventuelle entre pluviométrie et états du milieu a été analysée, ces derniers étant comparés entre eux et jugés à travers le comportement des plantes.

\section{Etats du milieu}

L'état hydrique instantané du sol sur les différentes placettes a été caractérisé par la confection de « coupes hydriques » (JAILLARD et CABIDOCHE, 1984). Outre la difficulté d'installation de matériel important sur les exploitations, nous avons préféré cette méthode à l'humidimétrie neutronique, peu fiable et trop peu précise dans les sols à argile gonflante. Les "coupes" consistent à effectuer des sondages à la tarière sur un transect de $3,2 \mathrm{~m}$ (soit 2 billons) tous les $0,25 \mathrm{~m}$ et, pour chacun des points du transect, tous les $0,10 \mathrm{~m}$ jusqu'à $0,80 \mathrm{~m}$ de profondeur (ou moins lorsque le calcaire est proche). Sur chaque échantillon, d'environ

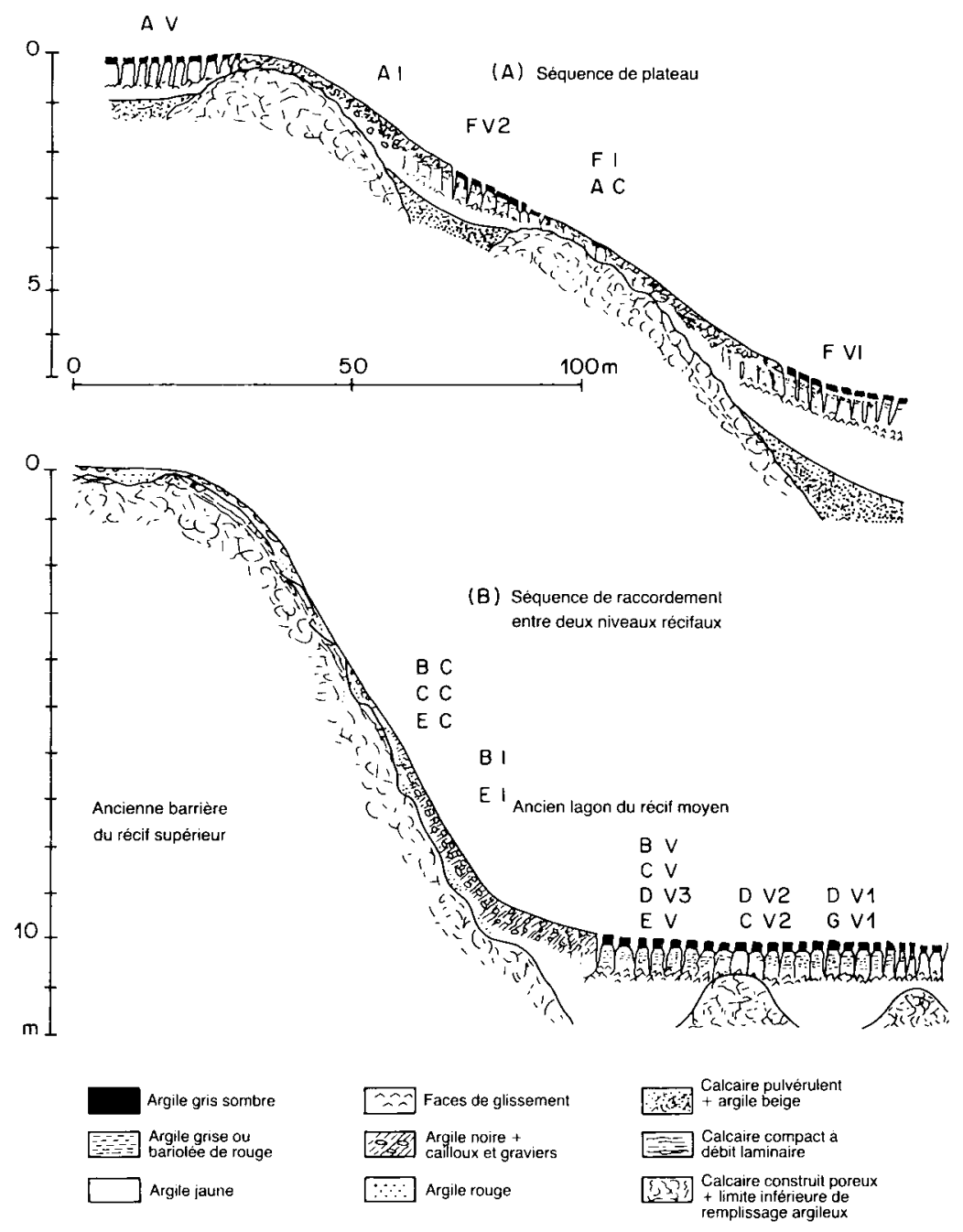

Figure 2

Position des placettes suivies sur des séquences type de sols à argile gonflante sur calcaire corralien (symboles du tabl. 3).

Block situation on typical sequence of swelling-clay soils developed on coral-reef limestone (symbols, see table 3 ). 
TABLEAU 1

Mode de conduite des parcelles.

Plot management.

\begin{tabular}{|c|c|c|c|c|c|c|}
\hline Parcelles & $\begin{array}{l}\text { Surface } \\
\text { (ares) }\end{array}$ & $\begin{array}{l}\text { Travail du sol } \\
\text { (date) }\end{array}$ & Fertilisation & Plantation & Densité & Problèmes \\
\hline A & 19 & $\begin{array}{l}\mathrm{L}-\mathrm{L}=13 \mathrm{j} \\
\mathrm{L}-\mathrm{S}=3 \mathrm{j}\end{array}$ & $\begin{array}{r}2 \text { apports } \\
160-80-120\end{array}$ & 12.08 & 20000 & - \\
\hline B & 24 & $\begin{array}{l}\mathrm{L}-\mathrm{L}=11 \mathrm{j} \\
\mathrm{L}-\mathrm{S}=1 \mathrm{j}\end{array}$ & $\begin{array}{r}2 \text { apports } \\
110-80-215\end{array}$ & 15.07 & 20000 & - \\
\hline $\mathrm{C}$ & 21 & $\begin{array}{l}\mathrm{L}-\mathrm{L}=12 \mathrm{j} \\
\mathrm{L}-\mathrm{S}=2 \mathrm{j}\end{array}$ & $\begin{array}{l}1 \text { apport } \\
110-0-0\end{array}$ & 28.08 & 20000 & (1) \\
\hline $\mathrm{D}$ & 10 & $\begin{array}{l}\mathrm{L}-\mathrm{L}=? \\
\mathrm{~L}-\mathrm{S}=?\end{array}$ & $\begin{array}{c}1 \text { apport } \\
120-80-240\end{array}$ & 21.07 & 18500 & - \\
\hline $\mathrm{E}$ & 19 & $\begin{array}{l}\mathrm{L}-\mathrm{L}=12 \mathrm{j} \\
\mathrm{L}-\mathrm{S}=1 \mathrm{j}\end{array}$ & $\begin{array}{l}2 \text { apports } \\
100-100-360\end{array}$ & 20.06 & 15600 & - \\
\hline F & 16 & $\begin{array}{l}L-L=15 j \\
L-S=9 j\end{array}$ & $\begin{array}{l}2 \text { apports } \\
100-100-360\end{array}$ & 12.08 & 16000 & - \\
\hline $\mathrm{G}$ & 10 & $\begin{array}{l}\mathrm{L}-\mathrm{L}=? \\
\mathrm{~L}-\mathrm{S}=?\end{array}$ & $?$ & 06.09 & 20000 & (2) \\
\hline
\end{tabular}

$\mathbf{L}-\mathbf{L}=$ nombre de jours entre les 2 labours.

$\mathrm{L}-\mathrm{S}=$ nombre de jours entre le deuxième labour et le sillonnage.

Problèmes : (1) importante attaque de Xanthomonas campesiris pv. vesicatoria Doidge,

(2) abandon.

TABLEAU 2

Doses d'arrosage et valeur de l'ETP pour les 3 premiers mois du cycle.

Rainfall, irrigation dose and PET for first three months.

\begin{tabular}{|c|c|c|c|c|c|c|c|}
\hline \multirow{2}{*}{ Placette } & \multicolumn{3}{|c|}{ Mois 1} & \multicolumn{2}{|c|}{ Mois 2} & \multicolumn{2}{|c|}{ Mois 3} \\
\hline & $I+P$ & ETP & $\mathrm{I}+\mathrm{P} / \mathrm{ETP}$ & $\mathbf{I}+\mathrm{P}$ & ETP & $\mathrm{I}+\mathrm{P}$ & ETP \\
\hline A V & 168 & \multirow{3}{*}{150} & 1,1 & 145 & \multirow{3}{*}{137} & 83 & \multirow{3}{*}{136} \\
\hline A I & 229 & & 1,5 & 238 & & 148 & \\
\hline $\mathrm{AC}$ & 192 & & 1,3 & 173 & & 195 & \\
\hline B V & 110 & \multirow{3}{*}{155} & 0,7 & 179 & \multirow{3}{*}{150} & 106 & \multirow{3}{*}{137} \\
\hline B I & 112 & & 0,7 & 145 & & 89 & \\
\hline B C & 167 & & 1,1 & 152 & & 106 & \\
\hline $\mathrm{CV}$ & 238 & \multirow{2}{*}{138} & 1,7 & 68 & \multirow{2}{*}{140} & 93 & \multirow{2}{*}{128} \\
\hline $\mathrm{C} \mathrm{C}$ & 250 & & 1,8 & 65 & & 98 & \\
\hline D V1 & 150 & \multirow{3}{*}{155} & 1,0 & 130 & \multirow{3}{*}{150} & 95 & \multirow{3}{*}{137} \\
\hline D V2 & 131 & & 0,8 & 118 & & 70 & \\
\hline D V3 & 288 & & 1,9 & 208 & & 218 & \\
\hline E V & 233 & \multirow{3}{*}{147} & 1,6 & 199 & \multirow{3}{*}{155} & 95 & \multirow{3}{*}{150} \\
\hline E I & 166 & & 1,1 & 115 & & 104 & \\
\hline $\mathrm{E} \mathrm{C}$ & 166 & & 1,1 & 83 & & 87 & \\
\hline F I & 164 & \multirow{3}{*}{150} & 1,1 & 154 & \multirow{3}{*}{137} & 95 & \multirow{3}{*}{136} \\
\hline F V1 & 166 & & 1,1 & 136 & & 100 & \\
\hline F V2 & 183 & & 1,2 & 163 & & 90 & \\
\hline G VI & 163 & \multirow{2}{*}{150} & 1,1 & $?$ & & $?$ & \\
\hline G V2 & 185 & & 1,2 & $?$ & & $?$ & \\
\hline
\end{tabular}


$200 \mathrm{~cm}^{3}$ est calculée l'humidité pondérale. L'image de l'état hydrique est obtenue en traçant par interpolation les courbes d'iso-valeur d'humidité. Le volume important de l'échantillon autorise le tracé d'un iso-contour. $\mathrm{Si}$ les coupes ne renseignent pas sur la quantité d'eau disponible pour la plante, en raison de la grande hétérogénéité des sols à argile gonflante et de la faible longueur du transect, elles renseignent sur le stockagedéstockage de l'eau dans les différents horizons.

Ensuite, lors de la réhumectation du profil par arrosage, elles révèlent les éventuelles discontinuités porales verticales. En raison de la relation entre taille des pores et infiltration, au moins dans les vertisols proprement dits, toute discontinuité macroporale entraîne une accumulation d'eau signifiée par un changement brutal d'humidité, mis en évidence sur les coupes par la proximité des différents iso-contours. Une coupe hydrique a été réalisée sur chaque placette au moment de la floraison, soit un mois environ après plantation. Quelques coupes ont été faites peu avant la fin du cycle.

L'état poral a été caractérisé par la description de profils culturaux avant la plantation et en fin de cycle sur chacune des placettes. Les premiers avaient pour double objectif de connaître l'état structural avant plantation et de s'assurer de l'identité des profils issus d'un même itinéraire technique. Les seconds servent à vérifier la convergence entre état hydrique instantané en réhumectation et état macroporal.

\section{Mesures et contrôles sur la culture, révélateurs des états du milieu}

Les dates d'apparition des fleurs et le nombre de fruits apparus ont été enregistrés sur chacune des placettes. La date de floraison est un critère révélateur des conditions de milieu, notamment d'aération (DUTHION, 1973 ; FlOCKER et al., 1959 ; REY et COSTES, 1966). Le rendement global en poids de fruits commercialisable n'a pu être mesuré, la récolte étant trop étalée. Cependant, le nombre de fruits est fortement lié au rendement final (CAVICCHI \& SilvetTi, 1976 ; SingH \& SiNGH, 1980). L'état phytosanitaire a été suivi tout au long du cycle.

\section{RÉSULTATS}

\section{A. Etats du milieu pour différentes doses d'irrigation}

Les profils culturaux effectués immédiatement après le double passage de charrue à disques font apparaître invariablement dans les vertisols :

- une couche travaillée faite d'agrégats centi- à millimétriques très secs enchâssant des mottes plus humides et de plus grande taille. La proportion de ces mottes varie selon les profils et semble liée à l'intervalle de temps s'écoulant entre les 2 labours. Dans tous les profils cependant, on trouve au fond de l'horizon travaillé une couche plus ou moins épaisse de terre fine très sèche,

- un horizon sous-jacent de couleur jaune, de structure continue et de porosité très faible. Les massifs sont séparés par des fissures suivant un maillage d'un mètre environ,
- enfin, à leur interface, un fond de labour ondulé et lissé par place au fond des dépressions. Ces lissages sont répartis de façon équidistante dans le profil et correspondent au passage des disques. La continuité des fissures, présentes dans l'horizon non travaillé est rompue à ce niveau par oblitération.

Dans les sols lithomorphes, les lissages du fond de labour existent également mais sont rompus par place par la présence d'éléments grossiers. Dans toutes les parcelles suivies, le profil en sol lithomorphe apparaît toujours globalement plus sec.

La figure 3 présente les « coupes hydriques » effectuées sur vertisols de dépression, 1 mois après plantation, soit approximativement à la floraison. En regard figurent la pluviométrie cumulée pendant ce mois $(\mathrm{Pc})$ et celle des 10 jours précédant la « coupe ». Les figures 4 et 5 montre les « coupes » faites respectivement sur les vertisols de pente et les sols lithomorphes à la floraison et la figure 6 les « coupes » réalisées peu avant la fin du cycle, en vertisols de dépressions.

\section{B. Mesures sur la culture}

Le description des profils en fin de cycle montre que dans les vertisols, l'enracinement ne colonise pas l'interrang, très tassé, et ne pénètre pas au-delà du fond de labour. A ce niveau, les racines sont gainées de rouille dans quelques placettes situées dans les dépressions. Dans les sols lithomorphes, quelques racines pénètrent le calcaire. Les parties lissées du fond de labour constituent cependant un obstacle à leur pénétration.

La comparaison entre les dates de floraison (figure 7) permet de classer les placettes au sein d'une même parcelle. Cette analyse par parcelle est la seule possible compte tenu de la variabilité de l'âge des plants au moment du repiquage. En $\mathrm{A}$, la floraison (date à laquelle 50 p. 100 des plants ont fleuri) intervient tout d'abord sur les vertisols de dépressions $(20 \mathrm{j})$ puis sur les vertisols de pente $(27 \mathrm{j})$, enfin dans les sols lithomorphes $(37 \mathrm{j})$. En B, la placette située sur vertisol de pente fleurit en premier $(12 \mathrm{j})$, suivie par celle implantée sur vertisol de dépression (17 j). Dans la parcelle $F$, la placette située sur vertisol de pente fleurit nettement plus tôt que celles sises dans les dépressions. Sur sol lithomorphe, la floraison subit un retard important (plus de $30 \mathrm{j}$ ). Dans la parcelle $\mathrm{D}$ aux sols homogènes, $\mathrm{D} \mathrm{V} 3 \mathrm{a}$ un retard ( $30 \mathrm{j}$ contre $20 \mathrm{j}$ pour les 2 autres) très marqué.

La variabilité des rendements au sein d'une même parcelle est elle-même très importante. Le nombre moyen de fruits par plantes (tabl. 3) est toujours plus élevé dans les placettes situées sur vertisols de pente dans les parcelles aux sols hétérogènes $(\mathrm{A}, \mathrm{B}$ et $\mathrm{E})$. En A, cependant, les rendements ne sont pas significativement différents. Entre placettes situées sur vertisols de dépression ou sols lithomorphes, l'avantage va à l'une ou l'autre des situations selon les parcelles. Peu différents en $\mathrm{A}$ (13,0 et 13,5 fruits par plante respectivement pour les vertisols de dépression et les sols lithomorphes), les rendements sont légèrement plus élevés sur vertisol de dépression en $B(15,9$ contre 14,3$)$, le contraire en revanche en E $(10,4$ contre 12,9). Dans les parcelles situées sur vertisols, le rendement dépend soit 

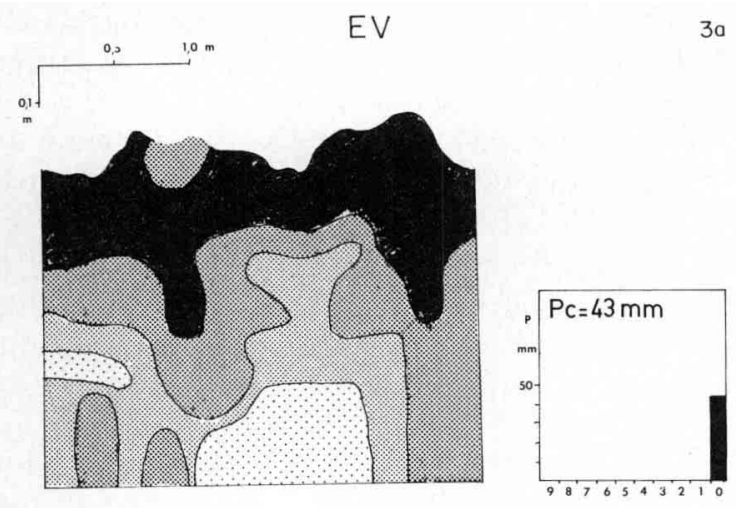

DV2
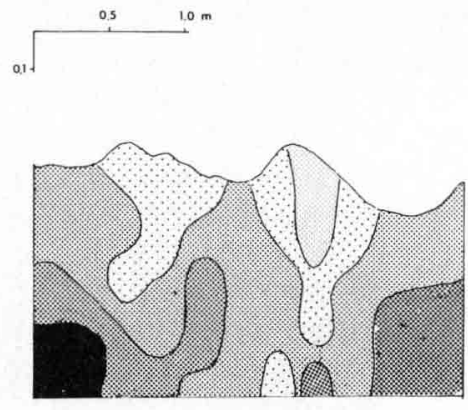

GV1

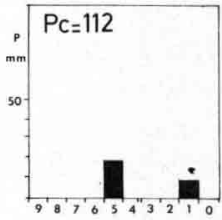

$3 f$

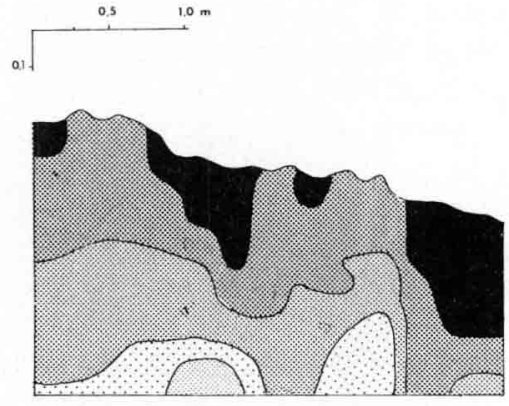

FV1

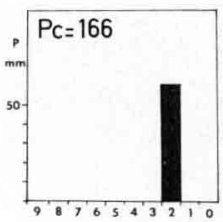

30
GV2

$3 c$
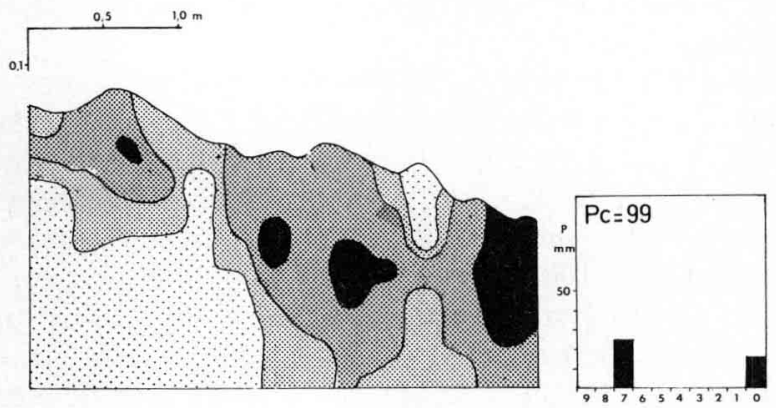

DV1

$3 d$

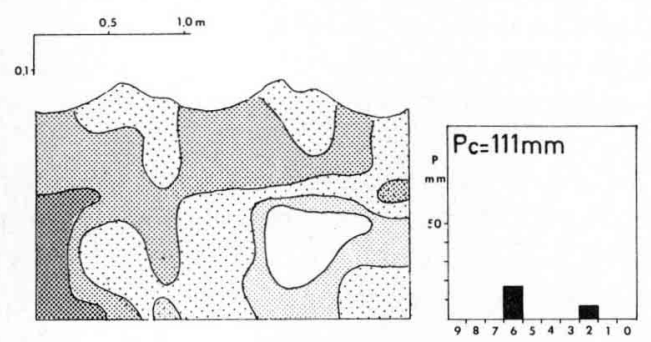

Figure 3

Coupes hydriques et pluviométrie en vertisols de dépressions à floraison de la culture.

Water content profile and rainfall in vertisols situated on depression at flowering.

Pc : Pluviométrie cumulée depuis le début du cycle.

Rainfall since begizning of cycle.

$J$ : Pluviométrie pendant les 10 jours précédant la coupe.

Rainfall during the 10 days before the water content profile.

L'échelle d'humidité est la même qu'en figure 1 .
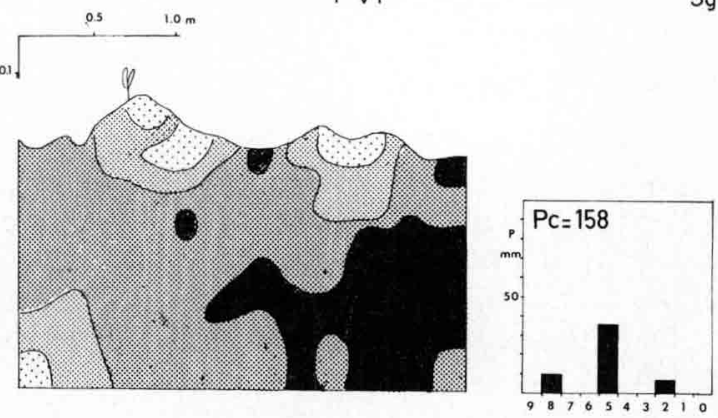

FV2

$3 \mathrm{~h}$
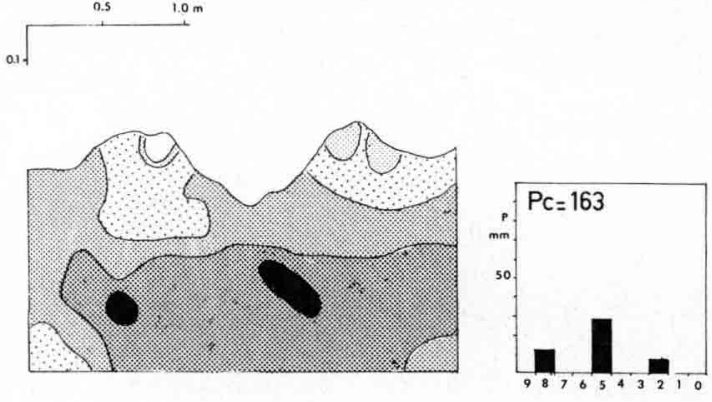

CV

$3 i$
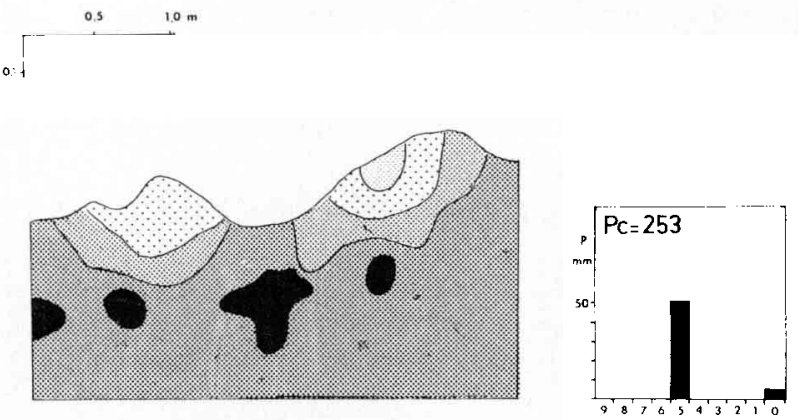
FI
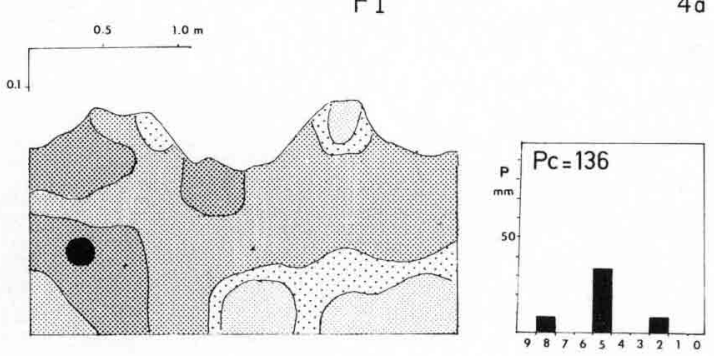

AI

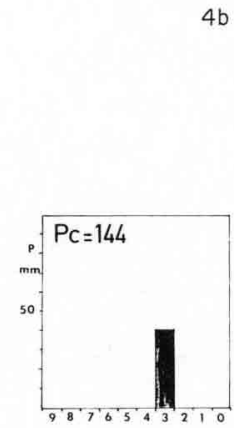

Figure 4

Coupes hydriques et pluviométrie en vertisols de pente à floraison. Water content profile and rainfall in vertisol at flowering.

DV2
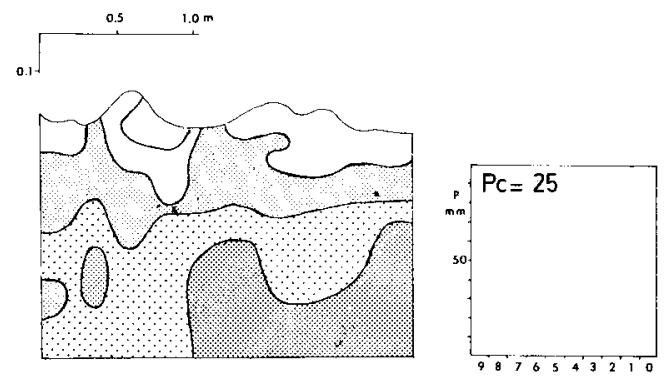

DV1

$6 b$
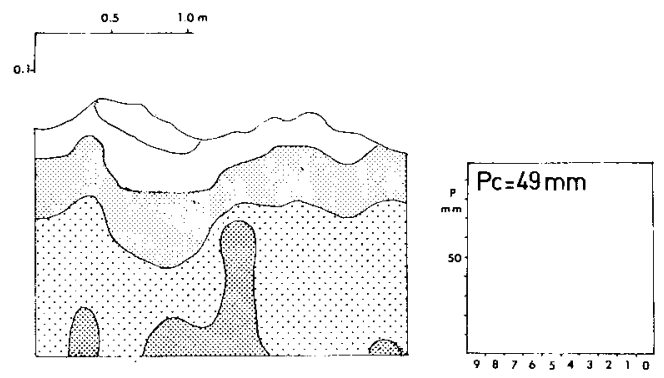

Figure 6

Coupes hydriques en vertisols de dépressions en fin de cycle. Water content profile in vertisols situated on depression at the end of the cycle.

Pc: Pluviométrie du mois précédant la coupe hydrique.

Rainfall during the month before the water content profile.
$A C$

$5 a$
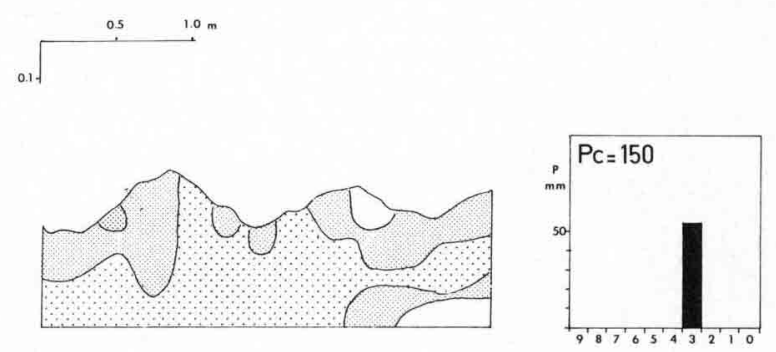

CC

$5 b$
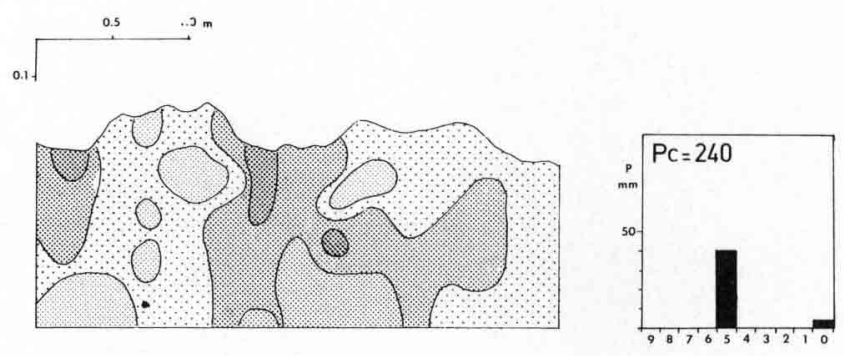

Figure 5

Coupes hydriques et pluviométrie en sols lithomorphes à floraison. Water content profile in shallow-stony soils at flowering.

TABLEAU 3

Nombre de fruits moyen par plante pour les différentes placettes. Average fruit number per plant at different sites.

\begin{tabular}{cccc}
\hline \multirow{2}{*}{ Parcelle } & Placettes & $\begin{array}{c}\text { Nombre de } \\
\text { fruits moyen }\end{array}$ & Ecart-type \\
\hline & A V & $13,0 \mathrm{a}$ & 6,1 \\
\cline { 2 - 4 } & A I & $15,8 \mathrm{a}$ & 5,7 \\
\cline { 2 - 4 } B & A C & $13,5 \mathrm{a}$ & 5,2 \\
\hline & B V & $15,9 \mathrm{a}$ & 9,9 \\
\hline & B I & $23,8 \mathrm{~b}$ & 9,1 \\
\hline \multirow{2}{*}{ D } & B C & $14,3 \mathrm{a}$ & 4,4 \\
\hline & D V1 & $11,3 \mathrm{a}$ & 4,7 \\
\hline \multirow{2}{*}{ E } & D V2 & $14,9 \mathrm{a}$ & 5,3 \\
\cline { 2 - 4 } & D V3 & $8,5 \mathrm{~b}$ & 3,8 \\
\hline & E V & $10,4 \mathrm{a}$ & 3,1 \\
\hline \multirow{2}{*}{ F } & E I & $16,1 \mathrm{~b}$ & 5,7 \\
\hline & E C & $12,9 \mathrm{a}$ & 6,1 \\
\hline & F I & $19,9 \mathrm{~b}$ & 5,3 \\
\hline & F V & $11,8 \mathrm{a}$ & 5,2 \\
\hline
\end{tabular}

Les chiffres suivis d'une même lettre ne sont pas significativement différents au seuil 0,05 au sein d'une même parcelle. 

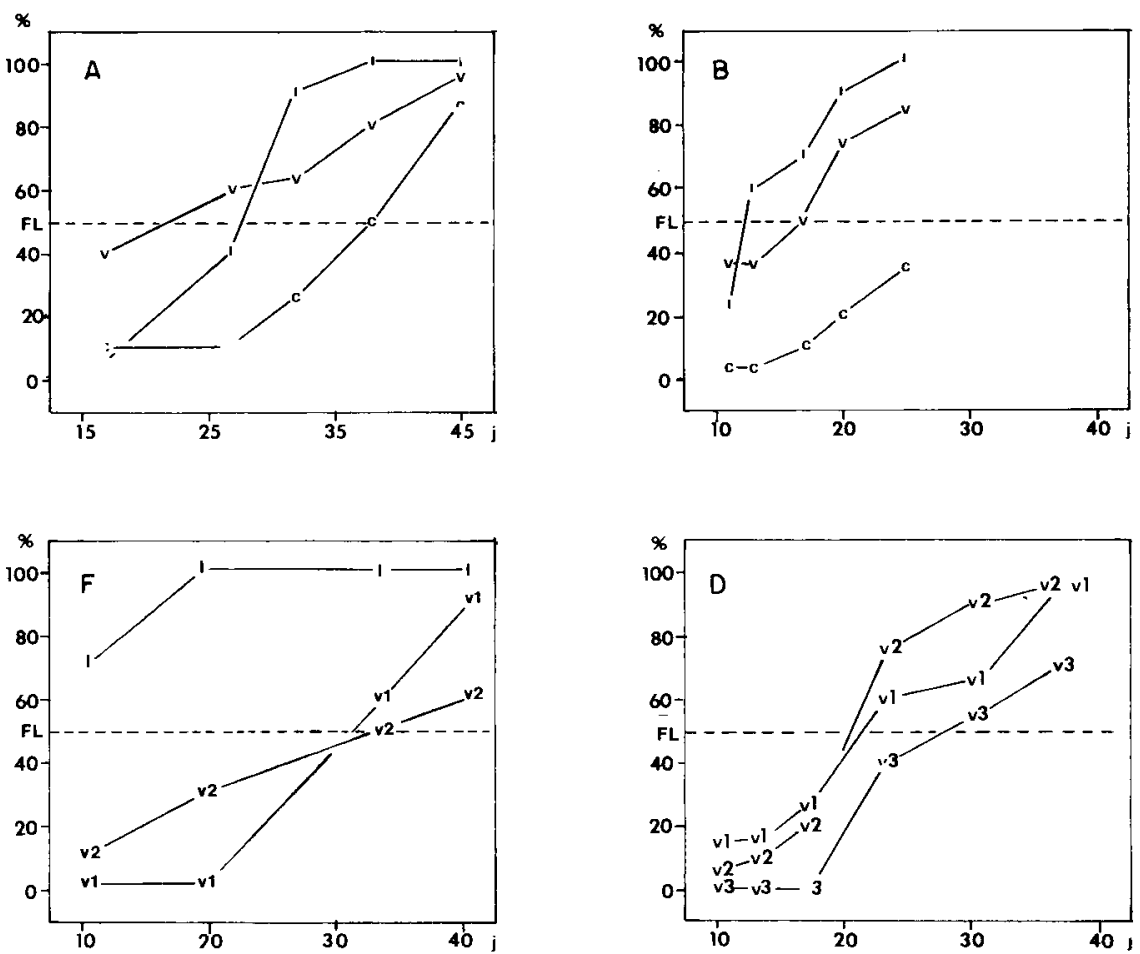

Figure 7

Pourcentage cumulé de plantes fleuries $\left(1^{\circ}\right.$ bouquet $)$ à une date don-

née.

Cumulated percentage of flowered plants.

Abscisse : nombre de jours entre plantation et floraison du premier

bouquet.

Horizontal axis : day after planting.

Ordonnée : pourcentage cumulé de plantes fleuries.

Vertical axis : cumulated percentage of flowered plants.

$F L$ : Date à laquelle 50 p. 100 ont fleuri.

$50 \%$ flowered plants.

$V$ : placettes situées sur vertisol de dépression (depression vertisol).

$I$ : placettes situées sur vertisol de pente (intermediate soil).

$C$ : placettes situées sur sol lithomorphe (shallow stony soil).

du régime pluviométrique (parcelle $\mathrm{D}$ ), soit de la topographie à régime pluviométrique équivalent (parcelle F). Les rendements de D V1 et D V2 sont significativement supérieurs à celui de $\mathrm{D}$ V3 où le régime pluviométrique est largement excédentaire. En F, à doses d'irrigation équivalentes excédentaires, les plantes de la placette située sur une convexité topographique (F I) produisent le plus grand nombre de fruits. Pour des raisons phytosanitaires, les rendements n'ont pas été mesurés sur 2 parcelles ( $\mathrm{G}$ et $\mathrm{C}$ ).

\section{DISCUSSION}

1. Dans les vertisols, les coupes hydriques suggèrent le rôle du fond de labour dans la limitation du drainage interne

Les coupes hydriques effectuées dans les vertisols de dépression montrent que seule la couche travaillée est affectée par le stockage et la consommation d'eau, et que la dynamique hydrique des horizons profonds et de l'interface avec la couche travaillée dépend du régime hydrique imposé :
- seules les irrigations très rationnées (fig. $3 b$ ) permettent une relative homogénéisation de l'ensemble du profil en un état " sec »; cependant, seule la couche travaillée se réhumecte de manière homogène après irrigation. Les horizons sous-jacents montrent une réhumectation hétérogène (fig. $3 a$ ). Immédiatement après irrigation (fig. $3 a$ et $3 c$ ), des îlots surhumectés (W > 0,6 g/g), qui jalonnent régulièrement le fond de labour, peuvent apparaître ;

- pour les irrigations rationnées (fig. $3 d$ et $3 e$ ), les horizons non travaillés conservent la forte hétérogénéité caractéristique des sols naturels. Quant à la couche travaillée, elle montre un fonctionnement disjoint, caractérisé par une humidité plus homogène. Cette indépendance fonctionnelle, au plan hydrique, de la couche travaillée n'est illustrée que par les très forts gradients verticaux d'humidité qu'expriment les resserrements des courbes d'isohumidité au voisinage du fond de labour ;

- quant aux irrigations proches de l'ETP (fig. $3 f$, $3 g$ et $3 h$ ), le résultat dépend des fréquences d'irrigation et des doses élémentaires apportées. En fortes doses très espacées (fig. $3 f$ ), la forte hétérogénéité du sol profond subsiste, avec humectation périfissurale visible par le resserrement horizontal des courbes d'iso- 
humidités ; la couche travaillée est relativement homogène et discordante par son humidité élevée, sur le sol non travaillé.

A l'inverse, en faibles doses rapprochées (fig. $3 g$ et $3 h$ ), l'hétérogénéité du sol profond s'estompe. L'humidité atteint des valeurs proches de la saturation « fentes fermées » sous savane spontanée ( $\mathrm{W}=0,55 \mathrm{~g} / \mathrm{g})$; les dessiccations sont limitées à la portion de la couche travaillée située sous les sommets de billons ; bien que l'on soit éloigné des irrigations fortes, les îlots surhumectés jalonnant le fond de labour subsistent. L'ensemble de ces phénomènes deviennent caricaturaux pour les irrigations nettement excédentaires (fig. $3 i$ ).

Ces faits sont compatibles avec un ralentissement de l'infiltration par le fond de labour qui obture les fentes. Ce ralentissement est décelable lors des réhumectations par le très fort gradient d'humidité sur la transition couche travaillée/sol profond. Même sous une irrigation rationnée, des îlots d'humidité très forte peuvent apparaître, jalonnant le fond de labour avec la même période que les gouttières lissées observées sur les profils culturaux. La rémanence de ces îlots est d'autant plus forte que l'on va vers les irrigations excédentaires. Les horizons non travaillés finissent alors par être réhumectés par diffusion pour être portés à une humidité proche de la saturation. Ils perdent ainsi l'hétérogénéité hydrique consécutive à l'infiltration fissurale.

L'accumulation d'eau est absente ou sporadique sur les vertisols de pente (fig. $4 a$ et $4 b$ ) ; si la rupture fonctionnelle introduite par le fond de labour est encore parfois visible par le resserrement vertical des courbes d'isohumidité (partie droite de la fig. $4 a$ ), on peut penser que l'effet de barrière à l'infiltration n'aboutit pas à des surhumectations visibles en raison du drainage latéral, que permet leur position topographique.

Enfin, dans les sols lithomorphes, on observe généralement une homogénéisation des teneurs en eau. Le contraste entre couche travaillée et sol profond s'atténue (fig. $5 a$ ). Même dans le cas d'une forte irrigation, on n'observe pas d'indépendance fonctionnelle de la couche travaillée. La présence de cailloux permet d'atténuer l'effet de barrière du fond de labour. Les profils montrent enfin une humidité globale beaucoup plus faible, traduction de la faible capacité de rétention en eau, et de la possibilité de drainage que présentent les sols caillouteux.

2. L'arrêt de l'enracinement sur cette discontinuité limite les possibilités de dessèchement des horizons non travaillés et empêche la réouverture des fentes dans les vertisols

On peut supposer que la formation rapide d'une nappe perchée sur le fond de labour dans les vertisols travaillés et irrigués à l'excès circonscrit le développement du système racinaire à la seule couche travaillée. Le défaut d'oxygénation du milieu, dû à l'ennoiement du fond de la couche travaillée, expliquerait les gaines de couleur rouille entourant les racines à ce niveau. L'absence de racines dans les horizons non travaillés limite fortement leur dessèchement et l'ouverture de fissures. Les travaux de RiTCHIE et BURNETT (1971) montrent, sur des sols ayant les mêmes caractéristiques (Houston black clay, Udic Pellusterts), que l'évaporation d'un sol nu est étroitement liée à l'humidité des
3 premiers centimètres. Lorsque celle-ci est inférieure à $0,20 \mathrm{~g} / \mathrm{g}$, clle se situe entre 0,25 et $0,50 \mathrm{~mm}$ par jour. Le dessèchement rapide de la couche travaillée et l'absence de racines rendent impossible le dessèchement profond et, donc, le retour au fonctionnement dual « fentes/infiltration rapide » - « massifs/diffusion lente » des vertisols sous savane spontanée. Malgré des arrosages très faibles en fin de cycle, la saturation des horizons profonds persiste (fig. 6).

\section{La différenciation de comportement hydrique des} vertisols et des sols lithomorphes entraîne l'hétérogénéité du développement et des rendements de la culture sur une même parcelle

La relation entre la pluviométrie et le comportement de la culture montre la supériorité des vertisols de pente par rapport aux autres types de sols pour un arrosage donné (tabl. 3). On peut supposer que le faible taux d'éléments grossiers leur confère une capacité de rétention de l'eau satisfaisante et un drainage latéral propre à éviter l'accumulation de l'eau. Sur les parcelles aux sols hétérogènes, lorsque l'arrosage est inférieur à ETP (B), les plantes situées sur les sols lithomorphes accusent un retard à la floraison et un rendement moindre. Il est probable que l'irrigation ne couvre pas les besoins. Par contre lorsque les doses sont excédentaires (E), l'effet dépressif des excès d'eau réduit de manière significative les rendements dans les dépressions. Il en est de même si l'on compare les rendements de parcelles où ils sont seuls représentés (parcelle D). Les faibles différences dans les modes de conduite autorisent le rapprochement entre pluviométrie et rendements pour les vertisols de dépression. Une corrélation négative et hautement significative apparaît entre ces 2 variables (fig. 8).

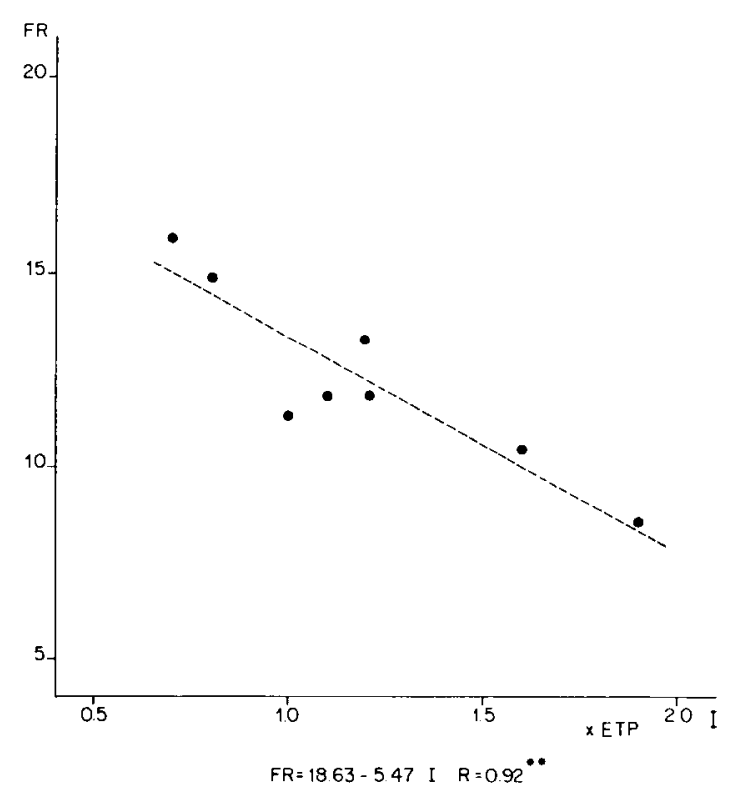

Figure 8

Rendements et pluviométries des placettes situées en vertisols de dépressions.

Yields and rainfall of the blocks situated on depression.

Abscisse : pluviométrie du premier mois du cycle. Horizontal axis : first month's rainfall.

Ordonnée : rendements en nombre de fruits par plantes. Vertical axis : average plant fruit number. 
Les travaux antérieurs d'ERICKSON \& VAN DOREN (1960) \& PERIGAUD S. (1967) montrent que tout défaut d'oxygénation, même de courte durée, peut avoir des conséquences néfastes sur le rendement. Le faible niveau d'aération de la base de la couche travaillée où se trouve la plus grande partie du système racinaire, noyé après chaque irrigation ou pluie, serait la cause de la chute des rendements dans ce type de sol.

\section{CONCLUSION}

L'irrigation dans les toposéquences de sols à argile gonflante sur calcaire corallien est délicate en raison de la variation de la profondeur des sols et de leur comportement sur de courtes distances. Peu drainants, mais présentant une capacité de rétention élevée lorsqu'ils sont profonds (vertisols), ils sont drainants mais séchants lorsque le taux d'éléments grossiers est élevé et que le calcaire perméable est proche (sols lithomorphes). Au sein d'une toposéquence, tous les intermédiaires de comportement existent entre ces deux extrêmes. Lorsqu'ils sont travaillés avec des outils obturant les fentes de retrait, les résultats obtenus sur une culture de tomate suggèrent le rôle du fond de labour dans l'affaiblissement du drainage interne des vertisols. Seule la couche travaillée participe au stockage-déstockage de l'eau, limitant les réserves disponibles. La fréquence des irrigations doit être accrue, mais le choix des doses à apporter demeure délicat. Tout arrosage excédentaire provoque l'accumulation de l'eau sur le fond de la couche travaillée et l'enn siement du système racinaire. En raison de l'arrêt des racines sur le fond de labour, provoqué par l'apparition précoce d'un milieu asphyxiant, les horizons non travaillés, une fois saturés n'évoluent plus et le retour à un fonctionnement proche de celui des vertisols en conditions naturelles assurant l'évacuation de l'eau en excès, compromis. On entrevoit ainsi la nécessité d'une jachère pluviale, provoquant, grâce à un enracinement dense et profond des espèces naturelles, un assèchement et donc une réouverture des fissures, permettant la restauration des possibilités de drainage des vertisols.

Ces conclusions découlant d'un constat de cohérence entre des hypothèses d'état poral et de remplissage de la porosité par l'eau et par l'air, et la réponse du peuplement végétal, méritent une confirmation expérimentale qui fait l'objet d'un prochain article.

Recu le 29 juillet 1986 Accepté le 5 janvier 1987.

\section{RÉFÉRENCES BIBLIOGRAPHIQUES}

Cabidoche Y. M., 1986. Distribution des sols à argiles gonflantes sur calcaire récifaux (zone Caraïbes). Utilisation de la résistivité électrique. Premier Séminaire scientifique de Pédologie pour la région de l'Amérique centrale et des Caraïbes. Sol et Eau, 8-20 avril 1985. Ed. ORSTOM, pp. 187-219.

Cavicchi S., Silvetti E., 1976. Yield in tomato. I - Multiple regression between yield and yield components. Genet. agrar., 30 (3/4), pp. 293-313.

Erickson A. E., Van Doren D. M., 1960. The relation of plant growth and yield to soil oxygen availability. 7th international congress of soil science, IV (54), pp. 428-434.

Duthion C., 1973. Etude des effets d'un excès d'eau sur les plantes. Exemple de la tomate. C. R. Acad. Sci., pp. 1461-1468.

Finck A., Venkateswarlu J., 1982. Chemical properties and fertility management of vertisols. In "Vertisols and rice soils in the tropics". Symposia paper II. I2th International congress of soil science. New Dehli, India, 8-16 feb. 1982, pp. 61-79.

Flocker W. J., Yomocil J. A., Howard F. D., 1959. Some responses of tomato to soil compaction. Soil Sci. Soc. Am. Proc., 23, pp. 188191.

Goss M. J., Ehlers W., Boone F. R., White I., Howse K. R., 1984. Effects of soil management practice on soil physical conditions affecting root growth. J. agric. Eng. Res., 30, pp. 131-140.

Goss M. J., Howse K. R., Harris W., 1978. Effects of cultivation on soil water retention and water use by cereals in clay soils. J. Soil Sci., 29 , pp. 475-488.

Jaillard B., Cabidochê Y. M., 1984. Etude de la dynamique de l'eau dans un sol argileux gonflant : dynamique hydrique. Sci. Sol $\mathrm{n}^{\circ} 3$, pp. 239-251.

Jewitt T. N., Law R. D., Virgo K. J., 1979. Vertisol soils of the tropics and sub-tropics. Their management and use. Outlook Agric., 10 (1), pp. 33-40.
Ney B., 1985. Evolution récente des systèmes de production et problèmes agronomiques nouveaux dans l'est de la Grande Terre de Guadeloupe. II - Les systèmes de culture dans les exploitations maraîchères du périmètre irrigué. Colloque « Systèmes de production agricoles caribéens et alternative de développement ". Martinique, 911 mai 1985. Ed. Université Antilles-Guyane, pp. 43-71.

Pellerin S., Ney B., 1985. Evolution récente des systèmes de production et problèmes agronomiques nouveaux dans l'est de la Grande Terre de Guadeloupe. I - Typologie des systèmes de production. Colloque "Systèmes de production agricoles caribéens et alternative de développement ». Martinique, 9-11 mai 1985. Ed. Université Antilles-Guyane, pp. 23-42.

Perigaud Simone, 1967. Effet du manque d'oxygène et de l'excès d'eau au niveau des racines sur la croissance et la nutrition globale de la plante. Ann. Agron., 18 (5), pp. 485-506.

Rey Y., Costes C., 1966. La physiologie de la tomate. Revue bibliographique. Doc. ronéoté I.N.R.A.

Ritchie J. T., Burnett E., 1971. Dryland evaporative flux in subhumid climate. II - Plant influences. Agron. J., 63 (1), pp. 56-61.

Schoch P. G., De Villele O., Granier J., 1975. Besoins climatiques en eau de cultures aux Antilles françaises. Nouv. agron. AntillesGuyane, 1-2, pp. 101-106.

Singh R. R., Singh M. N., 1980. Correlation studies in tomato. Indian J. Agric. Sci., 50 (8), pp. 595-598.

Virmani S. M., Sahrawat K. L., Burford J. R., 1982. Physiological and chemical properties of vertisols and their management. In "Vertisols and rice soils in the tropics". Symposia paper II. 12th International congress of soil science. New Dehli, India, 8-16 feb. 1982, pp. 80-93.

Warkentin B. P., 1982. Clay soil structure related to soil management. Trop. Agriculture, 59 (2), pp. 82-91. 